\section{SATELLITE SYMPOSIUM ABSTRACTS}

\author{
ROCHE SATELLITE SYMPOSIUM \\ High-Sensitivity Troponin Assays in Clinical Diagnostics, Under the Light of \\ Current Scientific Guidelines
}

Bulent Gorenek

Eskisehir Osmangazi University Cardiology Department, Eskisehir, Turkey

Chest pain is one of the most common reasons for an emergency room visit and it can be caused by a number of life-threatening conditions, including acute coronary syndromes (ACS).

Unstable angina, acute non-ST elevation myocardial infarction (NSTEMI), and acute ST elevation myocardial infarction (STEMI) are the three presentations of ACS. The first step in the management of patients with ACS is prompt recognition

Symptoms, cardiac biomarkers and 12-lead ECG should be evaluated together in diagnosis, risk stratification, and treatment of patients with suspected ACS. Cardiac troponins are widely used as one of the most important components of the ACS diagnosis. The troponin complex regulates the contraction of striated muscles and consists of three subunits (troponin $\mathrm{C}$, troponin $\mathrm{T}$, and troponin I). Their measurement can be performed using sensitive or high sensitivity tests. High sensitivity troponin assays should be preferred, when available. They have the ability to rapidly rule in or rule out ACS with improved sensitivity, and they are increasingly being used

A negative high-sensitive cardiac troponin T (hs-cTnT) test has a high negative predictive value, and may thus serve as an exclusionary test early in the diagnostic process. On the other hand, serial testing, as well as clinical context, are likely to become increasingly important for the interpretation of hs-cTnT assay results. Please note, many cardiac pathologies other than ACS also result in cardiomyocyte injury and, therefore, cardiac troponin elevations. Thus, it is important to understand how to interpret hscTn results.

\section{BECTON DICKINSON SATELLITE SYMPOSIUM}

Blood Sampling Approach from Intravenous Catheters and Children with International Guidelines and Literature Findings

Nedim Albayrak

Becton Dickinson, Dubai, UAE

Vascular access devices (VAD) are used to gain access to a patient's bloodstream through the veins to deliver a wide range of treatments, including vascular access for infusion therapy, parenteral nutrition, medication delivery, and blood draws. Although nurses may collect blood samples from peripheral intravenous catheters to reduce the number of needle sticks that patients must endure, this practice is associated with greater hemolysis. Some clinical exceptions include patients receiving thrombolytic agents or patients at increased risk of bleeding or possibly in an emergency situation with limited vascular access. Standardization of practice and education of staff will help minimize the equipment and technical factors associated with greater hemolysis. However, strategies must be developed to overcome barriers to changing this practice, including personal preference and feasibility. Therefore, we will focus on real-world evidence and some international guidelines recommendations to mitigate the risk of hemolysis. We will focus on WHO guidelines in the same session, especially the chapter discussing pediatric and neonatal blood sampling. Anyone taking blood from children and neonates must be well trained and practiced in venepuncture techniques. Additional focus is to discuss an effective way to apprehensive child into a cooperative patient.

\section{MINDRAY SATELLITE SYMPOSIUM}

The Great Value of Clinical Laboratories in the Pandemic Process

Sergio Bernardini

Tor Vergata University of Rome, Italy

Laboratory Medicine has been showing its great Value during Covid-19 pandemic in the whole care process: diagnosis, screening, follow up and outcome of the disease. The diagnostic tools to date are able to dose viral RNA, and antibodies against viral proteins or viral proteins themselves.

Even if the conventional RT-PCR has been the most widely used method, the greatest innovation in molecular diagnosis was reported by the so called CRISPR Community.

The first serological tests to be introduced were rapid serological tests with direct reading (first generation) or with fluorescence reading (2nd generation) and microfluidic with fluorescent reading (3rd generation).

Then conventional CLIA method have been introduced with better sensitivity and more recently a new kind of serological assay has been proposed able to detect anti SARS-Cov-2 serum antibodies throughout a competitive streptoavidin/biotin assay which utilize RBD as coated antigen and ACE-2 labelled with biotin as competitor molecule for serum antibodies.

Until few months ago the harmonization between many methods produced by many manufacturers was impossible. Recently the WHO produced an international standard (pool of sera-total antibodies) able to permit a sort of harmonization.

To date NGS return to be widely utilized in the effort to intercept new variants Finally, pathophysiology and natural course of Post-acute Sequelae of COVID-19 (PASC) also called "Long Covid"is unclear, meriting further studies. Also in this almost still unknown field, Clinical Laboratory could contribute in diagnosis and monitoring.

\section{ABBOTT SATELLITE SYMPOSIUM}

Clinical Decision Support System and Applications in the Laboratory

Umar Ansari

Abbott, Istanbul, Turkey

Umar Ansari has 16 years of experience in healthcare and informatics. In 2019, he top off his career by being deemed worthy of the "Top 100 Healthcare Leaders" award at the International Healthcare Advances (IFAH) forum. He continues to work on innovative solutions to overcome the challenges arising from factors such as digitalization, clinical decision support, telehealth and financial constraints of institutions.

We'll hear from him on how clinical and operational insights will impact health transformation and increase outcomes.

\section{SCIEX SATELLITE SYMPOSIUM}

New Mass Spectrometry Workflows and Fragmentation Pathways for The In-Depth Analysis of Complex Samples

Volker Kruft

SCIEX, Canada

Biological samples pose a number of challenges: they tend to contain a large number of analytes in widely varying concentrations, and the size of the sample sets to be analyzed can easily reach into the thousands.

Recent developments in the performance of high resolution qTOF instruments address these problems, the systems being very fast while maintaining high resolution. Acquisition rates exceeding $100 \mathrm{~Hz}$ in combination with dataindependent acquisition (DIA) routines allow the complete mapping in MS and $\mathrm{MS} / \mathrm{MS}$ of thousands of analytes.

At the same time, standard collision induced fragmentation (CID) in the mass spectrometer has long been recognized as not being sufficient to fully characterize all analytes; either not detecting critical attributes like the position of peptide modifications or not being strong enough to induce fragmentation, as is the case with many singly-charged peptides and isomeric metabolites. The recently introduced, tunable electron-activated fragmentation (EAD) addresses these needs. At the same time, the speed of EAD acquisition is in the range of 30 milliseconds, so does not slow down current high-speed mass spectrometry workflows.

Examples and results of all workflows and acquisition options will be presented.

\section{BINDING SITE SATELLITE SYMPOSIUM}

Monoclonal Gammopathies, Diagnosis and Monitoring

Mohannad Yacoub

Medical Science Liaison, International Sales, Birmingham, United Kingdom

Monoclonal gammopathies are a group of disorders characterized by the proliferation of a single clone of plasma cells. These plasma cells produce an immunologically homogeneous immunoglobulin, or parts thereof, also known as an M (for monoclonal) protein. We will be in a journey to discuss the Laboratory Methods for Diagnosis and monitoring of Monoclonal Gammopathies, focusing on clinical significance of FLC, HLC and MM panel and the usefulness to detect the remission and MRD's.

\section{SIEMENS PRESENTATION}

\section{Our Response to the COVID-19 Pandemic - Siemens Healthineers}

Fatih Kucukali

Siemens Healthineers, Istanbul, Turkey

The benefit of laboratory results and technology while determining the prognosis of the disease in Covid19 infection? Is it possible to increase the impact of the laboratory in clinical evaluation? By combining thousands of patient data with critical tests, we, as Siemens Healthineers, are shaping the future in an algorithm that works with machine learning and artificial intelligence. 